\title{
LOW FREQUENCY RESOLVENT ESTIMATES FOR LONG RANGE PERTURBATIONS OF THE EUCLIDEAN LAPLACIAN
}

\author{
JeAn-FranÇOIS Bony AND Dietrich HäFner
}

\begin{abstract}
Let $P$ be a long range metric perturbation of the Euclidean Laplacian on $\mathbb{R}^{d}, d \geq 3$. We prove that the following resolvent estimate holds:

$$
\left\|\langle x\rangle^{-\alpha}(P-z)^{-1}\langle x\rangle^{-\beta}\right\| \lesssim 1 \quad \forall z \in \mathbb{C} \backslash \mathbb{R},|z|<1,
$$

if $\alpha, \beta>1 / 2$ and $\alpha+\beta>2$. The above estimate is false for the Euclidean Laplacian in dimension 3 if $\alpha \leq 1 / 2$ or $\beta \leq 1 / 2$ or $\alpha+\beta<2$.
\end{abstract}

\section{Introduction}

There are now many results dealing with the low frequency behavior of the resolvent of Schrödinger type operators. The methods used to obtain these results are various: one can apply the Fredholm theory to study perturbations by a potential (see e.g. [7]) or a short range metric (see e.g. [9]). The resonance theory is also useful to treat compactly supported perturbations of the flat case (see e.g. [3]). Using the general Mourre theory, one can obtain limiting absorption principles at the thresholds (see e.g. [5] or [8]). The pseudo-differential calculus of Melrose allows to describe the kernel of the resolvent at low energies for compactifiable manifolds (see e.g. [6]). Concerning the long range case, Bouclet [2] has obtained a uniform control of the resolvent for perturbations in divergence form. We refer to his article and to [4] for a quite exhaustive list of previous results for perturbations of the Euclidean Laplacian.

On $\mathbb{R}^{d}$ with $d \geq 3$, we consider the following operator

$$
P=-b \operatorname{div}(G \nabla b)=-\sum_{i, j=1}^{d} b(x) \frac{\partial}{\partial x_{i}} G_{i, j}(x) \frac{\partial}{\partial x_{j}} b(x),
$$

where $b(x) \in C^{\infty}\left(\mathbb{R}^{d}\right)$ and $G(x) \in C^{\infty}\left(\mathbb{R}^{d} ; \mathbb{R}^{d \times d}\right)$ is a real symmetric $d \times d$ matrix. The $C^{\infty}$ hypothesis is made mostly for convenience, much weaker regularity could actually be considered. We make an ellipticity assumption:

$$
\exists C>0, \forall x \in \mathbb{R}^{d} \quad G(x) \geq C I_{d} \text { and } b(x) \geq C,
$$

$I_{d}$ being the identity matrix. We also assume that $P$ is a long range perturbation of the Euclidean Laplacian:

$$
\exists \rho>0, \forall \alpha \in \mathbb{N}^{d} \quad\left|\partial_{x}^{\alpha}\left(G(x)-I_{d}\right)\right|+\left|\partial_{x}^{\alpha}(b(x)-1)\right| \lesssim\langle x\rangle^{-\rho-|\alpha|} .
$$

In particular, if $b=1$, we are concerned with an elliptic operator in divergence form $P=-\operatorname{div}(G \nabla)$. On the other hand, if $G=\left(g^{2} g^{i, j}(x)\right)_{i, j}, b=\left(\operatorname{det} g^{i, j}\right)^{1 / 4}, g=$

Received by the editors October 5, 2009.

2000 Mathematics Subject Classification. 35P25, 47A10.

Key words and phrases. Resolvent estimates, asymptotically Euclidean manifolds. 
$\frac{1}{b}$, then the above operator is unitarily equivalent to the Laplace-Beltrami $-\Delta_{\mathfrak{g}}$ on $\left(\mathbb{R}^{d}, \mathfrak{g}\right)$ with metric

$$
\mathfrak{g}=\sum_{i, j=1}^{d} g_{i, j}(x) d x^{i} d x^{j},
$$

where $\left(g_{i, j}\right)_{i, j}$ is inverse to $\left(g^{i, j}\right)_{i, j}$ and the unitary transform is just multiplication by $g$.

Theorem 1. Let $P$ be of the form (1) in $\mathbb{R}^{d}$ with $d \geq 3$. Assume (H1) and (H2).

i) For all $\varepsilon>0$, we have

$$
\left\|\langle x\rangle^{-1 / 2-\varepsilon}(\sqrt{P}-z)^{-1}\langle x\rangle^{-1 / 2-\varepsilon}\right\| \lesssim 1,
$$

uniformly in $z \in \mathbb{C} \backslash \mathbb{R},|z|<1$.

ii) For all $\varepsilon>0$, we have

$$
\left\|\langle x\rangle^{-1 / 2-\varepsilon}(P-z)^{-1}\langle x\rangle^{-1 / 2-\varepsilon}\right\| \lesssim|z|^{-1 / 2},
$$

uniformly in $z \in \mathbb{C} \backslash \mathbb{R},|z|<1$.

iii) For all $\alpha, \beta>1 / 2$ with $\alpha+\beta>2$, we have

$$
\left\|\langle x\rangle^{-\alpha}(P-z)^{-1}\langle x\rangle^{-\beta}\right\| \lesssim 1,
$$

uniformly in $z \in \mathbb{C} \backslash \mathbb{R},|z|<1$.

Remark 2. $i$ ) The estimate (4) is not far from optimal. Indeed, this estimate is false for the Euclidean Laplacian $-\Delta$ in dimension 3 if $\alpha \leq 1 / 2$ or $\beta \leq 1 / 2$ or $\alpha+\beta<2$.

ii) One can interpret (4) in the following way: one needs a $\langle x\rangle^{-1 / 2}$ on the left and on the right to assure that the resolvent is continuous on $L^{2}\left(\mathbb{R}^{d}\right)$ and one needs an additional $\langle x\rangle^{-1}$ (distributed, as we want, among the left and the right) to guarantee that its norm is uniform with respect to $z$.

iii) By interpolation of (3) and (4), for $\alpha, \beta>1 / 2$ with $\alpha+\beta \leq 2$, one obtains estimates like (4) with $|z|^{-1+\frac{\alpha+\beta}{2}-\varepsilon}$ on the right hand side.

iv) In dimension 1 , the kernel of $(-\Delta-z)^{-1}$ is given by $\frac{i e^{i \sqrt{z}|x-y|}}{2 \sqrt{z}}$. In particular, this operator satisfies (3) but not (4) (for any $\alpha, \beta$ ). Therefore it seems that (3) is more general than (4). It could perhaps be possible to prove (3) in lower dimensions (at least, in dimension 2 and when $P$ is of divergence form $P=-\operatorname{div}(G \nabla)$ ).

$v$ ) For large $z$, the estimate (3) coincides with the high energy estimate in the non-trapping case. In particular, if we suppose in addition a non trapping condition for $P$, then (2) and (3) hold uniformly in $z \in \mathbb{C} \backslash \mathbb{R}$.

The proof of the above theorem is based on the low frequency estimates of [1]. Concerning the square root of $P$, they are used to treat the wave equation. Note that in [1] they are formulated for the Laplace-Beltrami operator $-\Delta_{\mathfrak{g}}$, but they obviously hold for the operators studied in the present paper. Essentially, we will show that $(2) \Rightarrow(3) \Rightarrow(4)$. 


\section{Proof of the results}

We begin by recalling some results of [1]. For $\lambda \geq 1$, we set

$$
\mathcal{A}_{\lambda}=\varphi(\lambda P) A_{0} \varphi(\lambda P),
$$

where

$$
A_{0}=\frac{1}{2}(x D+D x), \quad D\left(A_{0}\right)=\left\{u \in L^{2}\left(\mathbb{R}^{d}\right) ; A_{0} u \in L^{2}\left(\mathbb{R}^{d}\right)\right\},
$$

is the generator of dilations and $\varphi \in C_{0}^{\infty}(] 0,+\infty[;[0,+\infty[)$ satisfies $\varphi(x)>1$ on some open bounded interval $I=[1-\widetilde{\varepsilon}, 1+\widetilde{\varepsilon}], 0<\widetilde{\varepsilon}<1$ sufficiently small. As usual, we define the multi-commutators $\operatorname{ad}_{A}^{j} B$ inductively by $\operatorname{ad}_{A}^{0} B=B$ and $\operatorname{ad}_{A}^{j+1} B=$ $\left[A, \operatorname{ad}_{A}^{j} B\right]$. We recall [1, Proposition 3.1]:

Proposition 3. $i)$ We have $(\lambda P)^{1 / 2} \in C^{2}\left(\mathcal{A}_{\lambda}\right)$. The commutators $\operatorname{ad}_{\mathcal{A}_{\lambda}}^{j}(\lambda P)^{1 / 2}$, $j=1,2$, can be extended to bounded operators and we have, uniformly in $\lambda \geq 1$,

$$
\begin{aligned}
&\left\|\left[\mathcal{A}_{\lambda},(\lambda P)^{1 / 2}\right]\right\| \lesssim 1, \\
&\left\|\operatorname{ad}_{\mathcal{A}_{\lambda}}^{2}(\lambda P)^{1 / 2}\right\| \lesssim \begin{cases}1 & \rho>1, \\
\lambda^{\delta} & \rho \leq 1,\end{cases}
\end{aligned}
$$

where $\delta>0$ can be chosen arbitrary small.

ii) For $\lambda$ large enough, we have the following Mourre estimate:

$$
\mathbb{1}_{I}(\lambda P)\left[i(\lambda P)^{1 / 2}, \mathcal{A}_{\lambda}\right] \mathbb{1}_{I}(\lambda P) \geq \frac{\sqrt{\inf I}}{2} \mathbb{1}_{I}(\lambda P) .
$$

iii) For $0 \leq \mu \leq 1$ and $\psi \in C_{0}^{\infty}(] 0,+\infty[)$, we have

$$
\left\|\left\langle\mathcal{A}_{\lambda}\right\rangle^{\mu} \psi(\lambda P)\langle x\rangle^{-\mu}\right\| \lesssim \lambda^{-\mu / 2+\delta},
$$

for all $\delta>0$.

We will also need [1, Lemma B.12]:

Lemma 4. Let $\chi \in C_{0}^{\infty}(\mathbb{R})$ and $\beta, \gamma \geq 0$ with $\gamma+\beta / 2 \leq d / 4$. Then, for all $\delta>0$, we have

$$
\left\|\langle x\rangle^{\beta} \chi(\lambda P) u\right\| \lesssim \lambda^{-\gamma+\delta}\left\|\langle x\rangle^{\beta+2 \gamma} u\right\|,
$$

uniformly in $\lambda \geq 1$.

By Mourre theory (see Theorem 2.2 and Remark 2.3 of [1] for example) and Proposition 3 , we obtain the following limiting absorption principle:

$$
\sup _{\operatorname{Re} z \in I, \operatorname{Im} z \neq 0}\left\|\left\langle\mathcal{A}_{\lambda}\right\rangle^{-1 / 2-\varepsilon}\left((\lambda P)^{1 / 2}-z\right)^{-1}\left\langle\mathcal{A}_{\lambda}\right\rangle^{-1 / 2-\varepsilon}\right\| \lesssim \lambda^{\delta},
$$

for all $\varepsilon, \delta>0$. This entails the following

Lemma 5. For $\Psi \in C_{0}^{\infty}(] 0,+\infty[)$ and $\varepsilon>0$, we have

$$
\begin{aligned}
& \left\|\langle x\rangle^{-1 / 2-\varepsilon} \Psi(\lambda P)\left(\sqrt{P}-\lambda^{-1 / 2} z\right)^{-1}\langle x\rangle^{-1 / 2-\varepsilon}\right\| \lesssim 1, \\
& \left\|\langle x\rangle^{-1 / 2-\varepsilon} \Psi(\lambda P)\left(P-\lambda^{-1} z^{2}\right)^{-1}\langle x\rangle^{-1 / 2-\varepsilon}\right\| \lesssim \frac{\sqrt{\lambda}}{|z|},
\end{aligned}
$$

uniformly in $\lambda \geq 1$ and $z \in \mathbb{C} \backslash \mathbb{R}$ with $\operatorname{Re} z \in I$. 
Proof. Let $\widetilde{\Psi} \in C_{0}^{\infty}(] 0,+\infty[)$ be such that $\Psi \widetilde{\Psi}=\Psi$.

To prove the first identity, we write

$$
\begin{aligned}
\|\langle x\rangle^{-1 / 2-\varepsilon} & \Psi(\lambda P)\left(P^{1 / 2}-\lambda^{-1 / 2} z\right)^{-1}\langle x\rangle^{-1 / 2-\varepsilon} \| \\
\lesssim & \left\|\langle x\rangle^{-1 / 2-\varepsilon} \Psi(\lambda P)\left\langle\mathcal{A}_{\lambda}\right\rangle^{1 / 2+\varepsilon}\right\|\left\|\left\langle\mathcal{A}_{\lambda}\right\rangle^{-1 / 2-\varepsilon}\left(P^{1 / 2}-\lambda^{-1 / 2} z\right)^{-1}\left\langle\mathcal{A}_{\lambda}\right\rangle^{-1 / 2-\varepsilon}\right\| \\
& \times\left\|\left\langle\mathcal{A}_{\lambda}\right\rangle^{1 / 2+\varepsilon} \widetilde{\Psi}(\lambda P)\langle x\rangle^{-1 / 2-\varepsilon}\right\| \\
\lesssim & \lambda^{-\frac{1}{4}-\frac{\varepsilon}{2}+\delta} \lambda^{\frac{1}{2}+\delta} \lambda^{-\frac{1}{4}-\frac{\varepsilon}{2}+\delta} \lesssim 1 .
\end{aligned}
$$

Here we have used Proposition 3 iii), Lemma 4 as well as the fact that $\delta$ can be chosen arbitrary small.

To obtain (7), it is sufficient to write

$$
\begin{aligned}
\|\langle x\rangle^{-1 / 2-\varepsilon} & \Psi(\lambda P)\left(P-\lambda^{-1} z^{2}\right)^{-1}\langle x\rangle^{-1 / 2-\varepsilon} \| \\
\lesssim & \lambda^{1 / 2}\left\|\langle x\rangle^{-1 / 2-\varepsilon} \Psi(\lambda P)\left((\lambda P)^{1 / 2}+z\right)^{-1}\langle x\rangle^{1 / 2+\varepsilon / 2}\right\| \\
& \times\left\|\langle x\rangle^{-1 / 2-\varepsilon / 2} \widetilde{\Psi}(\lambda P)\left(P^{1 / 2}-\lambda^{-1 / 2} z\right)^{-1}\langle x\rangle^{-1 / 2-\varepsilon}\right\| \\
\lesssim & \frac{\lambda^{1 / 2}}{|z|} .
\end{aligned}
$$

Here we have used (6) and Lemma 4. It is clear from the proof of Lemma 4 in [1] that we can apply it to $\Psi(\lambda P)\left((\lambda P)^{1 / 2}+z\right)^{-1}$ and that we gain $\frac{1}{|z|}$. Indeed, as an almost analytic extension, we can just take the almost analytic extension of $\Psi$ multiplied by the analytic function $\frac{1}{\sqrt{x}+z}$.

Proof of Theorem 1. We only show the third part of the theorem, the proof of the other parts is analogous. Also it is clearly sufficient to replace $z$ by $\lambda^{-1} \widetilde{z}^{2}$ with $\operatorname{Re} \widetilde{z}=1 \in I$ and $\lambda \geq 1$ (for instance, $\lambda=(\operatorname{Re} \sqrt{z})^{-2}$ and $\widetilde{z}=\sqrt{z} /(\operatorname{Re} \sqrt{z})$ ). Let $\varphi, \widetilde{\varphi} \in C_{0}^{\infty}\left(\left[\frac{1}{3}, 3\right]\right)$ and $f \in C^{\infty}(\mathbb{R})$ be such that $\widetilde{\varphi}=1$ on the support of $\varphi, f(x)=0$ for $x<2$ and

$$
f(x)+\sum_{\mu=2^{n}, n \geq 0} \varphi(\mu x)=1,
$$

for all $x>0$. Since 0 is not an eigenvalue of $P$, we can write

$$
\begin{aligned}
\langle x\rangle^{-\alpha}(P-z)^{-1}\langle x\rangle^{-\beta}= & \langle x\rangle^{-\alpha} f(P)(P-z)^{-1}\langle x\rangle^{-\beta} \\
& +\sum_{\mu=2^{n}, n \geq 0}\langle x\rangle^{-\alpha} \varphi(\mu P)\left(P-\lambda^{-1} \widetilde{z}^{2}\right)^{-1}\langle x\rangle^{-\beta} .
\end{aligned}
$$

Of course, since $|z|<1$, the functional calculus gives

$$
\left\|\langle x\rangle^{-\alpha} f(P)(P-z)^{-1}\langle x\rangle^{-\beta}\right\| \lesssim 1 .
$$

Let $\widetilde{\alpha}=\min \left(\alpha, \frac{d}{2}\right)$ and $\widetilde{\beta}=\min \left(\beta, \frac{d}{2}\right)$. Note that $\widetilde{\alpha}+\widetilde{\beta}>2$ since $d \geq 3$. Let $\Psi \in C_{0}^{\infty}(] 0,+\infty[)$ be such that $\Psi=1$ near $\left[\frac{1}{12}, 12\right]$. Then, for $\frac{\mu}{4} \leq \lambda \leq 4 \mu$, we have

$$
\begin{aligned}
\|\langle x\rangle^{-\alpha} & \varphi(\mu P)\left(P-\lambda^{-1} \widetilde{z}^{2}\right)^{-1}\langle x\rangle^{-\beta} \| \\
\lesssim & \left\|\langle x\rangle^{-\alpha} \varphi(\mu P)\langle x\rangle^{1 / 2+\varepsilon}\right\|\left\|\langle x\rangle^{-1 / 2-\varepsilon} \Psi(\lambda P)\left(P-\lambda^{-1} \widetilde{z}^{2}\right)^{-1}\langle x\rangle^{-1 / 2-\varepsilon}\right\| \\
& \quad \times\left\|\langle x\rangle^{1 / 2+\varepsilon} \widetilde{\varphi}(\mu P)\langle x\rangle^{-\beta}\right\| \\
\lesssim & \lambda^{\frac{1}{4}+\frac{\varepsilon}{2}-\frac{\tilde{\alpha}}{2}+\delta} \lambda^{\frac{1}{2}}|\widetilde{z}|^{-1} \lambda^{\frac{1}{4}+\frac{\varepsilon}{2}-\frac{\tilde{\beta}}{2}+\delta} \lesssim \lambda^{1+\varepsilon+2 \delta-\frac{\tilde{\alpha}+\tilde{\beta}}{2}} \lesssim 1,
\end{aligned}
$$


for all $\varepsilon, \delta>0$ small enough. Here we have used (7) and two times Lemma 4 . On the other hand, for $\lambda \notin\left[\frac{\mu}{4}, 4 \mu\right]$, the functional calculus and Lemma 4 yield

$$
\begin{aligned}
\left\|\langle x\rangle^{-\alpha} \varphi(\mu P)\left(P-\lambda^{-1} \widetilde{z}^{2}\right)^{-1}\langle x\rangle^{-\beta}\right\| & \lesssim\left|\mu^{-1}-\lambda^{-1}\right|^{-1}\left\|\langle x\rangle^{-\alpha} \varphi(\mu P)\right\|\left\|\widetilde{\varphi}(\mu P)\langle x\rangle^{-\beta}\right\| \\
& \lesssim\left|\mu^{-1}-\lambda^{-1}\right|^{-1} \mu^{-\frac{\tilde{\alpha}+\widetilde{\beta}}{2}+\varepsilon},
\end{aligned}
$$

for all $\varepsilon>0$. Splitting the sum into two, we get

$$
\begin{gathered}
\sum_{4 \mu<\lambda}\left|\mu^{-1}-\lambda^{-1}\right|^{-1} \mu^{-\frac{\tilde{\alpha}+\tilde{\beta}}{2}+\varepsilon} \lesssim \sum_{4 \mu<\lambda} \mu \mu^{-\frac{\tilde{\alpha}+\tilde{\beta}}{2}+\varepsilon} \lesssim 1, \\
\sum_{\mu>4 \lambda}\left|\mu^{-1}-\lambda^{-1}\right|^{-1} \mu^{-\frac{\tilde{\alpha}+\tilde{\beta}}{2}+\varepsilon} \lesssim \sum_{\mu>4 \lambda} \lambda \mu^{-\frac{\tilde{\alpha}+\tilde{\beta}}{2}+\varepsilon} \lesssim 1 .
\end{gathered}
$$

This finishes the proof of the theorem. Note that the contribution of the energies less than $(4 \lambda)^{-1}$ can be estimated without dyadic decomposition.

Proof of Remark $2 i$ ). Let us recall that the kernel of the resolvent of the flat Laplacian in $\mathbb{R}^{3}$ at $z=0$ is given by

$$
K(x, y, 0)=\frac{1}{4 \pi|x-y|}
$$

Assume that $\langle x\rangle^{-\alpha}(-\Delta)^{-1}\langle x\rangle^{-\beta}$ is bounded on $L^{2}\left(\mathbb{R}^{3}\right)$. Applying to $\chi \in C_{0}^{\infty}\left(\mathbb{R}^{3}\right) \subset$ $L^{2}\left(\mathbb{R}^{3}\right)$, we find

$$
\left(\langle x\rangle^{-\alpha}(-\Delta)^{-1}\langle x\rangle^{-\beta} \chi\right)(x)=\int \frac{1}{4 \pi|x-y|}\langle x\rangle^{-\alpha}\langle y\rangle^{-\beta} \chi(y) d y \gtrsim\langle x\rangle^{-\alpha-1},
$$

for $|x| \gg 1$. But $\langle x\rangle^{-1-\alpha} \in L^{2}\left(\mathbb{R}^{3}\right)$ if and only if $\alpha>1 / 2$. The condition $\beta>1 / 2$ is checked in the same way. We now apply the resolvent to $f(x)=\langle x\rangle^{-3 / 2-\varepsilon} \in L^{2}\left(\mathbb{R}^{3}\right)$ and find

$$
\begin{aligned}
\left(\langle x\rangle^{-\alpha}(-\Delta)^{-1}\langle x\rangle^{-\beta} f\right)(x) & =\int \frac{1}{4 \pi|x-y|}\langle x\rangle^{-\alpha}\langle y\rangle^{-\beta}\langle y\rangle^{-3 / 2-\varepsilon} d y \\
& \geq \int_{|y| \leq \frac{|x|}{2}} \frac{1}{4 \pi|x-y|}\langle x\rangle^{-\alpha}\langle y\rangle^{-\beta}\langle y\rangle^{-3 / 2-\varepsilon} d y \\
& \gtrsim\langle x\rangle^{-\alpha-1} \int_{|y| \leq \frac{|x|}{2}}\langle y\rangle^{-3 / 2-\varepsilon-\beta} d y \gtrsim\langle x\rangle^{3 / 2-\alpha-\beta-1-\varepsilon} .
\end{aligned}
$$

This leads to the condition $2(3 / 2-\alpha-\beta-1) \leq-3$ which implies $\alpha+\beta \geq 2$.

\section{References}

[1] J.-F. Bony and D. Häfner, The semilinear wave equation on asymptotically Euclidean manifolds, Comm. Partial Differential Equations 35 (2010), no. 1, 23-67.

[2] J.-M. Bouclet, Low frequency estimates for long range perturbations in divergence form, arXiv:0806.3377.

[3] N. Burq, Décroissance de l'énergie locale de l'équation des ondes pour le problème extérieur et absence de résonance au voisinage du réel, Acta Math. 180 (1998), no. 1, 1-29.

[4] J. Dereziński and E. Skibsted, Classical scattering at low energies, in Perspectives in operator algebras and mathematical physics, Vol. 8 of Theta Ser. Adv. Math., 51-83, Theta (2008).

[5] S. Fournais and E. Skibsted, Zero energy asymptotics of the resolvent for a class of slowly decaying potentials, Math. Z. 248 (2004), no. 3, 593-633. 
[6] C. Guillarmou and A. Hassell, Resolvent at low energy and Riesz transform for Schrödinger operators on asymptotically conic manifolds. I, Math. Ann. 341 (2008), no. 4, 859-896.

[7] A. Jensen and T. Kato, Spectral properties of Schrödinger operators and time-decay of the wave functions, Duke Math. J. 46 (1979), no. 3, 583-611.

[8] S. Richard, Some improvements in the method of the weakly conjugate operator, Lett. Math. Phys. 76 (2006), no. 1, 27-36.

[9] X. P. Wang, Asymptotic expansion in time of the Schrödinger group on conical manifolds, Ann. Inst. Fourier 56 (2006), no. 6, 1903-1945.

Jean-François Bony, Institut de Mathématiques de Bordeaux, UmR 5251 du CNRS, Université de Bordeaux I, 351 cours de la Libération, 33405 Talence Cedex, France

E-mail address: bony@math.u-bordeaux1.fr

Dietrich Häfner, Université de Grenoble 1, Institut Fourier, UMR 5582 du CNRS, BP 74, 38402 St Martin D'Hères, France

E-mail address: Dietrich.Hafner@ujf-grenoble.fr 